\title{
DIGITAL TECHNOLOGY Investigating consultant-led virtual review as a model for implementing 7-day cardiology services in UK clinical practice
}

\author{
Authors: Alexander ] Deighton, ${ }^{\mathrm{A}}$ Ceri Davies, ${ }^{\mathrm{B}}$ Christos Bourantas, ${ }^{\mathrm{B}}$ Charles Knight, ${ }^{\mathrm{C}}$ Simon Woldman, ${ }^{\mathrm{D}}$ \\ James Deighton ${ }^{\mathrm{E}}$ Armita Azar $^{\mathrm{A}}$ and Debashish Das ${ }^{\mathrm{F}}$
}

\section{Background}

Disparities between weekend and weekday care, termed 'the weekend effect', have led to a UK government pledge to provide 7-day services. Despite this, poor outcomes have led to criticism of the programme. This study consequently sought to evaluate consultant-led virtual review as a model for 7-day cardiology services.

\section{Methods}

Over 4 weekends, cardiology patients underwent virtual review alongside in-person teams. Outcomes included length of stay, same-day discharge and 30-day mortality rates, as well as duration of ward rounds and change in patient management. Patients were surveyed on attitudes towards virtual review.

Results

Statistical analysis revealed no significant difference in clinical outcomes, while virtual review was noted to significantly decrease time taken $(p<0.0001)$. Attitudes towards virtual review were broadly favourable.

Conclusion

By demonstrating comparable outcomes compared with conventional review, as well as high acceptability, this study identified virtual review as an effective substitute for inperson care.

KEYWORDS: digital, cardiology, virtual, telemedicine, medicine

DOI: $10.7861 /$ fhj.2021-0115

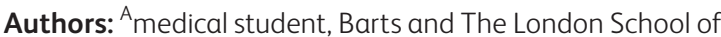
Medicine and Dentistry, London, UK; ${ }^{B}$ consultant cardiologist, The Royal Hospital, London, UK; ${ }^{C}$ consultant cardiologist and chief executive, St Bartholomew's Hospital, London, UK; ${ }^{\text {D }}$ consultant cardiologist and director, Barts Heart Centre, London, UK;

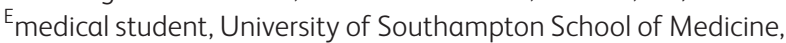
Southampton, UK; F consultant cardiologist, Whipps Cross University Hospital, London, UK

\section{Introduction}

Having analysed the medical records of almost four million patients admitted to Canadian hospitals between April and December 2001, Bell and Redelmeier first demonstrated 'the weekend effect', the objective fall in patient outcomes following weekend admission. ' Since then, this effect has been observed over a range of acute and chronic conditions, with large scale meta-analyses confirming a significant difference in overall weekend and weekday mortality rates. ${ }^{2}$ Despite uncertain aetiologies, this suggestion of a weekend effect has quickly gained political traction in the UK. Policy makers, including the secretary of state for health, have since responded with the promise of increased weekend service provision so as to produce a more consistent quality of care. This decision, supported by the Royal College of Physicians, has led to the establishment of the NHS Services, Seven Days a Week Forum, aimed at improving access to diagnostic, urgent and emergency services across the week. $^{3}$

While an ongoing process, however, broad criticisms of the 7-day programme remain. Proposed evidence supporting the need for 7-day services (7DS) has been quickly disputed and many, including The King's Fund, have questioned whether such a project is affordable in the current context, particularly with a lack of senior level practitioners to implement it. ${ }^{4,5}$ The capacity of general practice to deliver 7DS was further questioned by the Royal College of General Practitioners who argued that focus should be put on improving core services, as opposed to expanding access beyond them. ${ }^{6}$ Consequently, existing evidence for improved outcomes as a result of 7DS is conflicting and many academics believe the costly redistribution of already limited medical resources will outweigh any benefits to public health.

Unsurprisingly, these financial constraints and a lack of meaningful progress has put even greater impetus on finding novel, cost-effective means of providing 7DS and it is here that digital services such as telemedicine may play a role. These technologies, defined as the use of telecommunications for medical management and diagnosis, offer a solution to many of the physical barriers affecting access to care. ${ }^{7}$ What's more, existing evidence on the impact of telemedicine services on clinical workloads, patient satisfaction and patient outcomes suggests high acceptability, cost-effectiveness and improvements in both mortality rates and length of stay (LOS). ${ }^{8}$ 
Consequently, by implementing a process of senior-level virtual review in addition to existing protocols, this system has the means to streamline existing workflows, and their mutual development would surely be useful in the transformation of healthcare to meet the demands of 7DS. Yet, while there are published studies exploring telemedicine for patient review, there are currently no studies utilising these technologies to expand access to senior decision makers across the weekend. As a result, this study seeks to explore the feasibility and acceptability of consultant-led virtual review as a model for implementing 7-day cardiology services across UK clinical practice.

\section{Methods}

Study objectives

We formulated three research questions to be explored in this study.

> What is the effect of consultant-led virtual review on clinical patient outcomes?

> What are the attitudes of patients towards consultant-led virtual review?

> What are the organisational impacts of implementing consultant-led virtual review?

\section{Study design}

This study was implemented as a prospective, non-randomised cluster trial. Patients assigned to the control arm received conventional in-person consultant-led review, while patients assigned to the intervention arm were managed with remote consultant-led virtual review. Patients were assigned to each arm based on logistical suitability, in which those on wards with a higher quality internet connection were prioritised for virtual review. No other variables influenced group allocation. Included patients were followed up for 30 days via review of electronic patient records (EPR).

\section{Patient selection}

Eligible patients were over 18 years old with legal capacity and under the care of the cardiology team at Whipps Cross University Hospital. Patients referred for in-patient cardiology review were also considered eligible. Those who did not wish to participate, were unable to give consent or were in need of urgent intervention were not included in the study.

\section{Materials}

A written nine-item questionnaire was constructed to allow patients to rate aspects of the virtual review. The questionnaire included two items on the effectiveness of the consultation, one item on prior experience of telemedicine and six items on attitudes towards virtual review. In order to maximise accessibility, this questionnaire was provided to patients in both paper-based format and digitally using anonymised Google Forms.

\section{Videoconferencing system}

The smartphone-based videoconferencing system selected for the virtual review was the WhatsApp encrypted video-conferencing service, providing a two-way audio-visual connection with the reviewing cardiology consultant. Patient privacy was ensured by encrypting data before transmitting it via internet uplink. No video or audio data was stored following the consultation.

\section{Study protocol}

Included patients were reviewed at Whipps Cross University Hospital between November 2020 and February 2021. Following admission to the cardiology ward, or to the acute assessment unit (AAU), eligible patients were referred for either conventional or virtual consultant-led review as part of the daily ward round. This was undertaken alongside the same in-person cardiology team (one cardiology ST3-level specialty registrar, one foundation year-1 doctor and one medical student) who undertook necessary clinical examinations and relayed this to the reviewing consultant via the audio-visual feed. Each group was reviewed by the same consultant cardiologist and subsequent care pathways were identical. Patient records were accessed remotely via Cerner PowerChart. Alterations in diagnosis and management were relayed directly to the cardiology team and documented in patient notes.

\section{Outcome measures}

The primary outcome measures used in this study included LOS, need for patient transfer to a tertiary cardiology centre, same-day discharge and 30-day mortality rates, as well as organisational factors (time taken to complete review, whether there was a change in management following virtual review and whether there was a need for an in-person consultation following virtual review). Change in management was defined as change in medication, change in dose or change in prescribed therapies. Secondary outcome measures describe patient attitudes towards consultantled virtual review. The independent variables included patient demographic data extracted from electronic patient records.

\section{Statistical analysis}

Independent t-tests compared demographic characteristics and primary outcome measures between each study group. Chisquared tests were used to assess group differences in categorical measures. All statistical tests were two-sided, and all hypotheses were evaluated at the $5 \%$ significance level, with $p<0.05$ considered statistically significant. Items measuring attitudes towards consultant-led virtual review, and need for in-person follow-up underwent descriptive analysis.

\section{Results}

Between November 2020 and February 2021, a total of 53 patients were enrolled in the study: 32 patients in the virtual review arm and 21 patients in the control arm. No patients declined to participate, with baseline demographic characteristics provided in Table 1. In the virtual arm, 16 patients were admitted with new evidence of acute coronary syndrome, eight prior to elective surgical procedures, six with evidence of heart failure and two with significant arrhythmia. In the control arm, this amounted to 11, five, four and two patients, respectively. Statistical analysis demonstrated no significant difference in patient age, gender or rates of prior hospitalisation between the study arms. 
Table 1. Baseline characteristics and clinical outcomes of patients assigned to virtual review and control arms

\begin{tabular}{|c|c|c|c|c|}
\hline Characteristics & Control arm, $n=21$ & Virtual arm, $n=32$ & Standard deviation & $\mathrm{p}$-value \\
\hline Mean age, years & 68.1 & 71.5 & 11.811 & 0.996 \\
\hline Male, $\%$ & 76.2 & 56.3 & 0.321 & 0.139 \\
\hline Previous hospital admission, \% & 80.9 & 90.6 & 0.452 & 0.309 \\
\hline Mean length of stay, days & 14.1 & 9.9 & 2.654 & 0.054 \\
\hline Same-day discharge, \% & 0.0 & 16.6 & 0.306 & 0.063 \\
\hline 30-day mortality, \% & 4.8 & 0.0 & 0.144 & 0.396 \\
\hline Transfer to tertiary care centre, $\%$ & 0.0 & 6.3 & 0.245 & 0.512 \\
\hline Encounters resulting in change in management, \% & 95.2 & 76.6 & 0.373 & 0.062 \\
\hline Mean time taken per encounter, minutes & 14.4 & 4.8 & 1.727 & $<0.001$ \\
\hline Encounters resulted in need for in-person follow-up, \% & $N / A$ & 0.0 & $N / A$ & N/A \\
\hline
\end{tabular}

\section{Effect of virtual review on patient outcomes}

After a 30-day follow-up of patients, both clinical and organisational outcomes were compared between the two study arms, summarised in Table 1. LOS, calculated from the day of first review (on admission) until the day of discharge, was found to be lower in the virtual review group compared with the control group (9.9 vs 14.1 days). Similar results were reported for patient mortality. Here, 30 -day mortality rates were $4.8 \%$ for the control group, while no deaths were reported in the 30 days following virtual review. When conducting data analysis, however, no statistically significant differences were detected in LOS, need for institutional transfer or 30-day mortality rates between the control and virtual review groups ( $p=0.054, p=0.512$ and $p=0.396$, respectively).

Of the 68 patient encounters over the course of the study ( 47 in the virtual review arm and 21 in the control arm), mean time taken to review each patient was 4.8 minutes and 14.4 minutes for the virtual review and control groups, respectively. Technical difficulties were experienced prior to 10 virtual encounters, with the majority due to a poor internet connection resulting in inadequate audio and visual quality. These patients consequently did not go through the virtual review process. Eight of these encounters were successfully completed in-person, with the patient subsequently reassigned to the control arm. For the remaining two encounters, connection was able to be re-established within 5 minutes and the review was successfully completed. Time to reconnection was included in the total time of the encounter, except for cases that were reassigned to the control arm. Of the 47 encounters that were completed virtually, none went on to require a further in-patient review, while 36 (76.6\%) of these encounters lead to a change in either treatment plan or diagnosis. Notably, three patients seen virtually had been previously missed for post-take review. Further analysis of this data found virtual review to produce a non-significant difference in rate of change of management compared with the control arm $(p=0.062)$. For time taken, however, virtual review was demonstrated to produce a large and statistically significant effect ( 4.9 vs 14.2 minutes; $p \leq 0.0001$ ) compared with conventional review methods.

\section{Patient attitudes towards virtual review}

Of those in the intervention arm, 31/32 (96.9\%) completed the questionnaire, with subsequent responses summarised in Table 2.
When asked on technical issues, $90.3 \%$ and $96.8 \%$ reported ease of communication and clear video feeds, respectively. On attitudes, patients overwhelmingly indicated being comfortable in talking to a screen, and over $90 \%$ reported having trust in the telemedicine system being utilised in this study. Lower scores were reported when patients were asked whether or not the consultation had influenced their diagnosis or management, however, over $95 \%$ of patients reported an appropriate level of privacy, and $100 \%$ of patients surveyed reported feeling satisfied with the telemedicine services provided.

\section{Table 2. Patient evaluation of the virtual review}

\section{service}

\begin{tabular}{|c|c|c|c|}
\hline & Yes, \% & No, $\%$ & Don't know, \% \\
\hline $\begin{array}{l}\text { Do you have prior } \\
\text { experience of telemedicine? }\end{array}$ & 71.0 & 29.0 & 0.0 \\
\hline $\begin{array}{l}\text { Were you able to } \\
\text { communicate effectively? }\end{array}$ & 90.3 & 6.5 & 3.2 \\
\hline $\begin{array}{l}\text { Was the consultant clearly } \\
\text { visible on screen? }\end{array}$ & 96.8 & 3.2 & 0.0 \\
\hline $\begin{array}{l}\text { Did you feel comfortable } \\
\text { talking to a screen? }\end{array}$ & 93.5 & 0.0 & 6.5 \\
\hline Did you trust the system? & 96.8 & 0.0 & 3.2 \\
\hline $\begin{array}{l}\text { Was there an appropriate } \\
\text { level of privacy? }\end{array}$ & 96.8 & 0.0 & 3.2 \\
\hline $\begin{array}{l}\text { Do you think the } \\
\text { telemedicine process helped } \\
\text { in terms of your diagnosis } \\
\text { and treatment? }\end{array}$ & 83.9 & 0.0 & 16.1 \\
\hline $\begin{array}{l}\text { Are you satisfied with the } \\
\text { telemedicine service you } \\
\text { were given today? }\end{array}$ & 100.0 & 0.0 & 0.0 \\
\hline $\begin{array}{l}\text { Would you recommend } \\
\text { this to others in a similar } \\
\text { position to yourself? }\end{array}$ & 93.5 & 0.0 & 6.5 \\
\hline
\end{tabular}




\section{Discussion}

With telemedicine being increasingly adopted across both the UK and globally, there is a clear need for further research and evaluation into which modalities are used, and where in the clinical pathway telemedicine is best suited. This continues to be the case for out-of-hours and weekend care, in which research is still limited despite the potential for cost-savings and improved clinical outcomes. Consequently, this study explores the impact of a virtual-review programme on clinical outcomes and patient acceptability.

\section{Principal findings}

In exploring the impact of this programme, we were able to demonstrate comparable outcomes between virtual review and conventional means of healthcare provision. For instance, no statistically significant differences were observed between the two study arms in LOS, mortality rate and need for patient transfer. Similarly, no appreciable difference was noted in those undergoing a change in management following either virtual or in-person review, while no patients in the virtual arm were found to require in-person follow-up.

These results, alongside a significantly reduced time taken to complete review, highlight the potential of virtual programmes as a cost-effective substitute for conventional review. Notably, the reduction in time taken to complete virtual review ( 4.8 vs 14.4 minutes per patient; $p<0.0001$ ) may reflect the ability of the virtual consultant to review patient notes when moving between bays, eliminating this time from the review process itself. Several key observations were also made about the benefits of the virtual review programme. Patients who had been missed for post-take review, for example, were seen during the morning ward round by the consultant, calling in via the virtual review platform. This prevented the need for general medicine physicians to follow up such patients on the cardiology ward, and allowed these patients to receive earlier and more specialised cardiology review. Due to the reduced consultant cover over the weekend, the same level of consultant access was not available in those treated conventionally. Similarly, those in the intervention arm could be seen more frequently due to virtual review, and those who required interpretation of real-time echocardiography or other imaging modalities while on the ward received rapid input from the virtual consultant, who was able to access previous diagnostic imaging via patient portals and review live images through the audio-visual feed. Had the consultant not been present, as is often the case in weekend care, this real-time interpretation and input would not have been possible, and such patients would certainly have had to wait longer for specialist input and decision making.

Following review, when provided with written questionnaires, the majority of patients reported effective communication and clear visibility, and felt the process was well organised. Fewer patients (83.9\%) felt the consultation had impacted their management or diagnosis. This, however, may reflect limited communication with the patient regarding clinical decisions, or a broader lack of medical understanding rather than ineffective consultation.

Overall, $100 \%$ of patients surveyed stated they were satisfied with the virtual review service. Such results are supported by findings from similar surveys, demonstrating high levels of satisfaction across a range of telehealth and tele-rounding services, even in older patients who may be considered more digitally averse. ${ }^{9,10}$ It may be, however, that the clinician-driven nature of the virtual review service allows for improved patient satisfaction in this age group, with further studies indicating comparable acceptability to younger adults. ${ }^{11}$

\section{Barriers to virtual review}

Despite these outcomes, a number of key barriers were identified in the implementation of virtual review programmes, the most prominent of which related to internet connectivity. Various areas of the hospital, such as the acute assessment unit, were deemed to have too poor a connection to provide consistent, high quality review, resulting in difficulty reaching the consultant or poor audio-visual communication. One review was interrupted by an incoming call on the handheld device, highlighting a possible need for dedicated devices set aside for this purpose. This mirrored the results of previous studies on the barriers to telemedicine uptake in which problems were faced with clarity and speed of networks. ${ }^{12}$ Interestingly, similar connectivity issues were identified in the control arm, due to the increasing reliance on EPR and digital communications. More broadly, consideration must be given to the security and appropriate encryption of patient data. This may prioritise audio rather than video-conferencing due to greater ease of encryption, while patients with sensory and/or cognitive impairment such as mutism and hearing/visual difficulty may require the aid of additional technologies or human resources. ${ }^{13}$ Significantly, however, due to use of existing computer stations, handheld devices and free-to-download video-conferencing software, there were no upfront costs associated with this service, eliminating cost as a barrier to virtual review.

\section{Research significance}

With the virtual review demonstrating equivalent or improved outcomes compared with conventional care, there may be potential for more widespread uptake of telemedicine technologies in out-of-hours care where there is greater need for consultant cover and cost-effective means of extending core services. If established effectively, greater access to senior clinical decision makers provides an opportunity to improve quality of secondary care, as well as lowering costs by increasing the efficiency of the review process, reducing bed pressures and preventing unnecessary interventions. This aligns with work done by both Delgoshaei et al and MacKinney et al who demonstrated significant net savings through the implementation of hospital telemedicine programmes. ${ }^{14,15}$ At the same time, there is significant scope to improve patient wellbeing during inpatient stays, although care must be taken to select the most patientfriendly platforms if this is to be achieved.

\section{Limitations and future research}

In carrying out this work, we have identified limitations in our study design. Primarily, with a small sample size, the study yields less statistical power. Non-randomised selection, based on logistical suitability, raises potential for selection bias, while patients were not analysed under an intention-to-treat (ITT) model. Respondents to the patient questionnaire were required to read and understand English, thus excluding non-English speakers and those who had poor literacy levels. Furthermore, without measuring baseline health status prior to receiving the 
intervention, differences in disease severity between the two study arms cannot be ruled out. For this reason, subsequent studies would benefit from larger cohorts, greater randomisation, an ITT analysis, and the collection of additional baseline demographics, coding data and clinical measurements (such as National Early Warning Scores (NEWS) and the Charlson Comorbidity Index).

\section{Conclusion}

With its increasing accessibility, and use alongside electronic patient records, telemedicine and virtual review have the potential to provide specialist input to out-of-hours and weekend services in which access to senior clinical decision makers is often lacking. While requiring further research, this claim is supported by the results of this study, whereby virtual review showed equal or greater clinical outcomes compared with conventional in-person consultation. Evidently then, with careful implementation, appropriate protection of patient data and by working to understand the attitudes of patients and staff, telemedicine has the potential to expand from community practice into both secondary and tertiary care settings.

\section{Acknowledgements}

Sincere thanks go to healthcare staff on Elizabeth Ward at Whipps Cross University Hospital for their kind assistance in carrying out this project.

\section{Conflicts of interest}

Alexander ] Deighton and Debashish Das report paid positions with Ortus-iHealth, a virtual outpatients company.

\section{References}

1 Bell CM, Redelmeier DA. Mortality among patients admitted to hospitals on weekends as compared with weekdays. N Engl J Med 2001;345:663-8.

2 Honeyford K, Cecil E, Lo M, Bottle A, Aylin P. The weekend effect: does hospital mortality differ by day of the week? A systematic review and meta-analysis. BMC Health Serv Res 2018;18:870.
3 NHS England. NHS Services, Seven Days A Week Forum: summary of initial findings. NHS, 2013.

4 Rimmer A, Kmietowicz Z. BM] editor writes to Hunt over misuse of weekend mortality data. BMJ 2015;351:h5624.

5 Appleby J. A 7/7 NHS: what price equity? BMJ 2016;352:1404.

6 Martens D. GPs challenge seven-day NHS proposal. CMAJ 2015; 188:E9.

7 Currell R, Urquhart C, Wainwright P, Lewis R. Telemedicine versus face to face patient care: effects on professional practice and health care outcomes. Cochrane Database Syst Rev 2000:CD002098.

8 Kohl BA, Fortino-Mullen M, Praestgaard A et al. The effect of ICU telemedicine on mortality and length of stay. J Telemed Telecare 2012;18:282-6.

9 Serhal E, Kirvan A, Sanches M, Crawford A. Client satisfaction and experience with telepsychiatry: development and validation of a survey using clinical quality domains. J Med Internet Res 2020;22:e19198.

10 Czaja SJ, Charness N, Fisk AD et al. Factors predicting the use of technology: findings from the Center for Research and Education on Aging and Technology Enhancement (CREATE). Psychol Aging 2006;21:333-52.

11 Mayworm AM, Lever N, Gloff N et al. School-based telepsychiatry in an urban setting: efficiency and satisfaction with care. Telemed J E Health 2020:26:446-54.

12 Acharya RV, Rai J]. Evaluation of patient and doctor perception toward the use of telemedicine in Apollo Tele Health Services, India. J Family Med Prim Care 2016;5:798-803.

13 Guinart D, Marcy P, Hauser M, Dwyer M, Kane JM. Mental health care providers' attitudes toward telepsychiatry: a systemwide, multisite survey during the COVID-19 pandemic. Psychiatr Serv 2021;72:704-7.

14 Delgoshaei B, Mobinizadeh M, Mojdekar R et al. Telemedicine: A systematic review of economic evaluations. Med J Islam Repub Iran 2017;31:113.

15 MacKinney AC, Ward MM, Ullrich F et al. The business case for teleemergency. Telemed J E Health 2015;21:1005-11.

Address for correspondence: Alexander J Deighton, Barts and The London School of Medicine and Dentistry, School of Medicine, Garrod Building, Turner Street, London E1 2AD, UK. Email: a.deighton@smd17.qmul.ac.uk Twitter: @AlexJDeighton 